\title{
Characterisation of the haematological profile, peripheral lymphocytes and cytokine production capacity of children with mild allergic asthma
}

\author{
E. Nova ${ }^{1}$, T. Pozo ${ }^{1}$, G. Garcia ${ }^{2}$, J. Romeo ${ }^{1}$, S. Gomez-Martinez $^{1}$, L. E. Diaz ${ }^{1}$, J. Warnberg $^{1}$, \\ A. A. Duarte ${ }^{1}$, A. Nogales ${ }^{2}$ and A. Marcos ${ }^{1}$ \\ ${ }^{1}$ Grupo de Inmunonutricion, Instituto del Frí-ICTAN, CSIC, Madrid, Spain and ${ }^{2}$ Pediatrics Department, Hospital Doce \\ de Octubre, Madrid, Spain
}

\begin{abstract}
Allergic asthma is a common immune-mediated disorder characterised by reversible airway inflammation, mucus production and variable airflow obstruction with airways hyperresponsiveness. Allergen exposure stimulates eosinophilic inflammation of the airways associated with infiltration of T-cells. Although activated memory CD4 + T cells have been involved in the pathogenic mechanisms of allergic asthma through production of Th2 cytokines (IL-4, IL-13) facilitating antigen-specific IgE production by B cells, other T cell types also accumulate in the airways and may play a role in the complex immune mechanisms of allergic asthma ${ }^{(1)}$. The relationship between infiltrated immune cells in the airways and circulating cells in the periphery is not clear. The aim of this study was to characterise the haematological laboratory values, the lymphocyte subsets and the cytokine production capacity of peripheral blood mononuclear cells (PBMCs) of children with allergic asthma.

Thirty-five children (aged 7-11 years) with intermittent or mild-persistent asthma (GINA classification, 2002) due to perennial allergy were recruited at the Hospital Doce de Octubre (Madrid). Fifty healthy children were also evaluated as a control group. Patients were asked to fill in a symptoms, medication and peak expiratory flow (PEF) diary (morning and evening) during the 2 weeks prior to blood extraction. Blood samples were used for blood cell count and differential analysis and for the immunophenotyping of the following lymphocyte subsets: $\mathrm{CD} 3+, \mathrm{CD} 4+, \mathrm{CD} 8+, \mathrm{CD} 4+\mathrm{CD} 45 \mathrm{RA}+, \mathrm{CD} 4+\mathrm{CD} 45 \mathrm{RO}+, \mathrm{CD} 8+\mathrm{CD} 45 \mathrm{RA}+, \mathrm{CD} 8+\mathrm{CD} 45 \mathrm{RO}+, \mathrm{CD} 3-$ $\mathrm{CD} 16+56+$ and CD19+ cells (four-colour, dual-laser Flow Cytometry, Becton Dickinson). One subject with suspected infection at blood collection was discarded from the analysis. PBMCs were isolated by Ficoll-Hypaque gradient centrifugation and cultured for $48 \mathrm{~h}$ with PHA $(7 \mu \mathrm{g} / \mathrm{ml})$ and LPS $(1.5 \mu \mathrm{g} / \mathrm{ml})$ to stimulate cytokine production. IFN- $\gamma$, TNF- $\alpha$, IL-10, IL- 6 , IL-4 and IL-2 were measured in culture supernatants by flow cytometry (CBA, BD Biosciences). Student's $t$ test was used for the statistical analysis.

Children with allergic asthma showed increased levels of RBC, haemoglobine, haematocrite and MCHC compared to the controls. WBC were similar in both groups, however, the percentage and counts of lymphocytes, monocytes, eosinophils and basophils was higher, while neutrophil percentage and counts were lower than in the control group. The percentage of CD3 +, CD4 +, CD8 + and CD19+ cells was also higher in asthmatic patients than in healthy subjects. This fact, together with the increased lymphocyte numbers $(P=0.030)$ resulted in significantly higher counts of the T and B lymphocyte subsets. On the contrary, NK $(C D 3-C D 16+56+)$ cell percentage was lower in asthma and absolute counts were similar in both groups. Naïve and memory helper and cytotoxic T cell percentages were similar to the control values. Regarding cytokine production, IFN- $\gamma$ and IL-10 were significantly lower in the asthmatic patients than in controls $(P=0.001)$, while IL-2 production was higher than in controls $(P=0.03)$.

These findings suggest that the neutrophil/lymphocyte ratio is decreased in children with mild allergic asthma and that the Th2 type cytokine production pattern that drives the pathogenic mechanisms of allergic asthma in the airways is partially present in peripheral blood T lymphocytes.
\end{abstract}

We acknowledge DANONE S.A for financial support.

1. Afshar R, Medoff BD \& Luster AD (2008) Clin Exp Allergy 38, 1847-1857. 